\title{
The Impact of Social and Clinical Complexity on Diabetes Control Measures
}

\author{
Erika K. Cottrell, PhD, MPP, Jean P. O'Malley, MPH, Katie Dambrun, MPH, \\ Brian Park, MD, MPH, Michelle A. Hendricks, PhD, Hongzhi Xu, PhD, MPH, MS, \\ Mary Charlson, MD, Andrew Bazemore, MD, MPH, Elizabeth Ann Shenkman, PhD, \\ Abby Sears, MHA, MBA, and Jennifer E. DeVoe, MD, DPhil
}

Purpose: In an age of value-based payment, primary care providers are increasingly scrutinized on performance metrics that assess quality of care, including the outcomes of their patient population in key areas such as diabetes control. Although such measures often adjust for patient clinical risk factors or clinical complexity, most do not account for the social complexity of patient populations, despite research demonstrating the strong association between social factors and health.

Metbods: Using patient electronic health record data from 2 large community health center networks serving safety net patients, we assessed the effect of both clinical and social risk factors on poor glucose control among diabetics. Logistic regression results were used to estimate the impact of adjusting for both clinical and social complexity on provider performance metrics. Clinical complexity was measured at the patient-level using the Charlson Comorbidity Index. Social complexity was measured at the community-level using the Social Deprivation Index.

Results: Clinical complexity alone was not consistently associated with poor diabetes control (ie, HbA1c $>9 \%$ ) in diabetic patients with HbA1c testing during the study period. However, increasing social complexity was significantly associated with higher rates of poor diabetic control in both cohorts. After adding adjustment for social complexity down to the national median score, our models suggest that approximately $25 \%$ of providers would have 1 to $2 \%$ improvement in the assessment of their diabetes control measures, with $45 \%$ showing a 2 to $5 \%$ improvement, and $5 \%$ showing more than a $5 \%$ improvement.

Conclusions: Providers caring for patients with greater social risk factors may benefit from having their performance metrics adjusted for the social complexity of their patient populations. $(\mathrm{J}$ Am Board Fam Med 2020;33:600-610.)

Keywords: Blood Glucose, Chronic Disease, Community Health Centers, Comorbidity, Diabetes Mellitus, Disease Management, Electronic Health Records, Glycated Hemoglobin A, Logistic Models, Patient Care, Primary Health Care, Risk Factors, Social Determinants of Health

\section{Introduction}

In an age of value-based payment, primary care providers are increasingly scrutinized on performance metrics that purport to measure quality of

This article was externally peer reviewed.

Submitted 11 October 2019; revised 14 February 2020; accepted 19 February 2020.

From OCHIN, Inc, Portland, OR (EKC, JPO, KD, MAH, AS); Department of Family Medicine. Oregon Health and Science University, Portland (EKC, JPO, BP, JED); University of Florida, Gainesville (HX, EAS); Cornell University School of Medicine, Ithaca, NY (MC); American Board of Family Medicine, Lexington, KY (AB).

Funding: The research reported in this publication was conducted using the National Patient-Centered Clinical Research Network (PCORnet $\left.{ }^{\circledR}\right)$. PCORnet ${ }^{\circledR}$ has been developed with funding from the Patient-Centered Outcomes care, including the health outcomes of their patient population and the extent to which their patients use services that increase costs, such as visits to the

Research Institute (PCORI). The study was funded by PCORI through PCORI Award (HSD-1603-34987).

Conflicting and Competing Interests: MC: Cornell University has filed a patent for the use of the enhanced comorbidity index to predict future costs. No other authors have conflicting or competing interests.

The views presented in this work are solely the responsibility of the author(s) and do not necessarily represent the views of organizations participating in, collaborating with, or funding PCORnet $^{\circledR}$ or of the Patient-Centered Outcomes Research Institute (PCORI).

Corresponding author: Erika Cottrell, $\mathrm{PhD}, \mathrm{MPP}, 1881$ SW Naito Pkwy, Portland, OR 97201. (E-mail: cottrelle@ ochin.org). 
emergency department. ${ }^{1-3}$ However, outcomes on many of these measures are influenced not only by provider actions, but also by patient behavior and comorbidity as well as social factors that impact their health. Indeed, a large body of research suggests that social determinants of health $(\mathrm{SDH})$ - "the conditions in which people are born, grow, live, work, and age"-play a powerful role in shaping health and contribute as much, or more, to population health than health care services. ${ }^{4,5}$ Patients with more social risk factors (eg, lower income, limited education, and residing in highly deprived neighborhoods) have increased health care needs, ${ }^{6}$ face greater obstacles in following medical recommendations, and are more vulnerable to poor health outcomes. ${ }^{7,8}$ Recent evidence suggests that performance metrics may depend on the SDH of a system's patient population, as much or more than patients' clinical complexities or individual provider performance..$^{9-13}$ As a result, providers who serve populations with more social risk factors-or greater social complexity-may seem to have lower performance metrics, relative to those serving less socially complex populations. ${ }^{14,15}$

Despite a growing body of evidence suggesting that adverse SDH can negatively impact the Triple Aim of improved health outcomes, better quality of care, and lower health care expenditures, few performance measures account for social complexity. ${ }^{16,17}$ Instead, the complexity of a patient or a panel of patients is largely determined by the number and type of clinical diagnoses and medical care utilization. ${ }^{18}$ This is explained in part by data limitations. While commonly used complexity indices accounting for clinical comorbidity can be computed from diagnosis codes, ${ }^{19}$ information on patients' SDH has traditionally been absent from most clinical and claims datasets. ${ }^{16,17}$ This is beginning to change and several recent national initiatives have highlighted the need for documenting a set of patientreported SDH domains in electronic health records (EHRs). ${ }^{20-23}$ However, there are numerous barriers to integrating $\mathrm{SDH}$ screening into clinical settings and ensuring standardized documentation in EHRs. ${ }^{24,25}$ As a result, despite recent increases in EHR documentation of patientreported social risks, it will take time before patientlevel SDH information is collected consistently across large populations of patients. ${ }^{26,27}$
Patient addresses, however, are routinely collected in EHRs and can be linked to publicly available datasets (eg, US Census, American Community Survey) that describe the social, economic, and environmental characteristics of the neighborhoods and communities where patients live. These area-level data-which some refer to as "community vital signs" - could be used as a proxy for patient-level SDH information, thus providing a readily available opportunity to explore the impact of social complexity on provider performance metrics. ${ }^{20,28}$ Using EHR data from 2 large networks of community health centers (CHCs), we worked closely with patient, provider, and health system stakeholders to assess the effect of patient-level clinical complexity and community-level social complexity on diabetes control, a prominent focus of performance measurement initiatives for over a decade. ${ }^{15,29,30}$ Our objectives were to 1) understand the relative contributions of clinical and social complexity to the probability of poor diabetes control, and 2) evaluate the association between patient clinical and social complexity and provider performance metrics. The results may have considerable implications for policy and payment, especially given increasing calls to shift toward models of value-based care. ${ }^{31}$

\section{Methods}

\section{Design and Setting}

This retrospective cohort study utilized EHR data extracted from OCHIN (not an acronym) and Health Choice Network (HCN), 2 large CHC networks serving safety net patients with high levels of social complexity. OCHIN provides a centralized instance of Epic EHR to over $600 \mathrm{CHCs}$, serving over 2 million patients, across 18 states. ${ }^{32,33} \mathrm{HCN}$ provides a centralized EHR for CHCs serving over 2 million patients across 19 states. Both are members of the National Patient-Centered Clinical Research Network (PCORnet)-OCHIN is part of the Accelerating Data Value Across a National Community Health Center (ADVANCE) network and $\mathrm{HCN}$ is part of OneFlorida-and thus have researchready EHR data formatted using the PCORnet common data model. The study was conducted in partnership between the OCHIN and the University of Florida and was approved by the Western Institutional Review Board and the University of Florida Institutional Review Board. 


\section{Study Sample}

The study sample included patients aged 18 to 75 years established in primary care clinics in the OCHIN or HCN network in 2015. Established patients were defined as those with an office visit in 2015 to a clinic and a prior visit (any year) to the same clinic. Among established patients, those with at least 1 outpatient encounter diagnosis of diabetes who received hemoglobin A1c (HbA1c) testing in 2015 were included in the analyses. Primary care providers with at least 20 established patients in the OCHIN or $\mathrm{HCN}$ network were included in the sample.

\section{Variables}

The primary dependent variable was diabetes control status; patients with HbA1c values greater than 9\% were considered poorly controlled. The threshold for diabetes control was chosen to align with the 2014 Physician Quality Reporting System Measure Specifications Manual for Claims and Registry Reporting of Individual Measures. ${ }^{34}$ If a patient had multiple HbA1c tests, the most recent result was used.

Independent variables included measures of patient-level clinical complexity and community-level social complexity. Patient-level clinical complexity was assessed using the Charlson Comorbidity Index (CCI), a validated measure that predicts the risk of mortality and resource utilization for patients with a range of comorbid conditions. ${ }^{35}$ The CCI has been used extensively to adjust for clinical complexity in health care utilization models. ${ }^{11,36,37}$ Because mental health conditions are strongly associated with health outcomes ${ }^{38}$ but were not included in the original CCI, we supplemented the score by adding an ordinal variable summarizing mental and behavioral health complexity (based on ICD-9 and ICD-10 diagnosis codes for depression, substance use disorders, and psychosis/bipolar disorders; see Appendix 1). Patient data from 2015 and all available data in years prior $^{1}$ were utilized to calculate the CCI.

To assess community-level social complexity, we utilized the social deprivation index score (SDI), a weighted composite measure of 7 indicators (percent living in poverty, percent with less than 12 years of education, percent single parent households, percent living in rented housing unit, percent living in overcrowded housing unit, percent of

\footnotetext{
${ }^{1}$ Patient data may extend as far back as $10 / 25 / 2005$, but differs from patient to patient depending upon data availability.
}

households without a car, and percent nonemployed adults under 65 years of age) developed using factor analysis. The SDI is calculated as a percentile ranking based on all Zip Code Tabulation Areas (ZCTA) and census tracts in the country. It has previously been shown to be associated with population-level health outcomes. ${ }^{39,40} \mathrm{We}$ used a version of the index that utilizes 2011 to 2015 American Community Survey 5-year estimates. Each patient in the sample was assigned an SDI score at the lowest level of geography possible, using available information on patient address. HCN patients were geocoded at the ZCTA-level. OCHIN patients were geocoded at the census tract level where possible, and at the ZCTA level if their census tract could not be determined.

\section{Analysis}

All analyses were conducted in parallel by OCHIN and University of Florida (for HCN) analysts, who utilized shared syntax, but did not share patientlevel data. Logistic regression models were computed to assess the odds of poor glucose control (HbA1c $>9 \%)$. Independent variables included the CCI (categorized as 0 to 1,2 to 3,4 to 5 , and $6+$ ), an additional variable to assess the number of mental/behavioral health diagnoses (categorized as $0,1,1+$; see Appendix 1), and SDI score (continuous variable with 1 unit increments equivalent to 10 National percentile ranks). Models were conducted in a stepped-wise fashion, with the first controlling for CCI, the second for CCI and mental/behavioral health diagnoses, and the third for CCI, mental/behavioral health diagnoses, and SDI score. Additional patient covariates were limited to those used by Centers for Medicare and Medicaid Services (CMS) in assessing provider quality metrics, which included age and gender.

Following the CMS procedure for adjustment of Performance Metrics ${ }^{41}$ for panel clinical complexity, we adjusted quality metrics for providers with more than 20 patients in the outcome. The denominator was determined from the formula: $\mathrm{P}_{\mathrm{adj}}=\left(\mathrm{P}_{\text {obs }} / \mathrm{P}_{\text {exp }} \times \mathrm{P}_{\text {pop }}\right.$ where $\mathrm{P}_{\text {adj }}$ is the percent of a provider's diabetic panel with poor glucose adjusted for model covariates, obtained by multiplying $\mathrm{P}_{\mathrm{pop}}$ (the observed percentage in the entire sample) by the ratio of $\mathrm{P}_{\text {obs }}$ (the observed panel percentage) to $\mathrm{P}_{\exp }$ (the percentage expected based on the providers patient panel). $\mathrm{P}_{\text {exp }}$ (the percentage expected based on the providers patient panel) was obtained by summing the predicted probabilities for their patients 


\begin{tabular}{|c|c|c|}
\hline & OCHIN & $\mathrm{HCN}$ \\
\hline Providers & 898 & 114 \\
\hline Patients & 63,906 & 9422 \\
\hline Poor glucose control (HbA1c greater than 9.0) & $14,021(21.9 \%)$ & $2374(25.2 \%)$ \\
\hline Female & $35,675(55.8 \%)$ & $5385(57.2 \%)$ \\
\hline \multicolumn{3}{|l|}{ Age } \\
\hline 12 to 21 & $347(0.5 \%)$ & $50(0.5 \%)$ \\
\hline 22 to 49 & $19,831(31.0 \%)$ & $2839(30.1 \%)$ \\
\hline 50 to 64 & $30,152(47.2 \%)$ & $5271(55.9 \%)$ \\
\hline 64 or older & $13,576(21.2 \%)$ & $1262(13.4 \%)$ \\
\hline \multicolumn{3}{|l|}{ Charlson Comorbidity Index } \\
\hline less than 2 & $31,554(49.4 \%)$ & $6625(70.3 \%)$ \\
\hline 2 to 3 & $21,852(34.2 \%)$ & $2073(22.0 \%)$ \\
\hline 4 to 5 & $7742(12.1 \%)$ & $375(4.0 \%)$ \\
\hline 6 or higher & $2758(4.3 \%)$ & $349(3.7 \%)$ \\
\hline \multicolumn{3}{|l|}{ Mental and Behavioral Health Diagnoses } \\
\hline 0 & $45,107(70.6 \%)$ & $6915(73.4 \%)$ \\
\hline 1 & $16,582(26.0 \%)$ & $2019(21.4 \%)$ \\
\hline$>1$ & $2217(3.5 \%)$ & $488(5.2 \%)$ \\
\hline Median SDI Score $\left(25^{\text {th }}\right.$ percentile, $75^{\text {th }}$ percentile $)$ & $79(55,92)$ & $80(63,91)$ \\
\hline
\end{tabular}

HBA1c, hemoglobin A1c; SDI, Social Deprivation Index; OCHIN, Oregon Community Health Information Network; HCN, Health Choice Network.

from the logistic regression model and multiplying the sum by 100 .

\section{Results}

\section{Sample Cohort Characteristics}

Table 1 provides sociodemographic and both social and clinical complexity characteristics for the study sample. The sample cohorts for diabetes outcomes included 63,906 OCHIN patients, $9422 \mathrm{HCN}$ patients, $898 \mathrm{OCHIN}$ providers and $114 \mathrm{HCN}$ providers. As might be expected among CHC patients, both the OCHIN and HCN cohorts had median SDI score that were higher than the national median (79 for OCHIN, 80 for HCN, compared with the national median of 50). The percentages of patients with HbAlc > 9\% (21.9\% for OCHIN patients and 25.2\% for $\mathrm{HCN}$ patients) were lower than those reported by the National Committee for Quality Assurance for 2015 (45.4\% for Medicaid patients, 27.4\% for Medicare Health Maintenance Organization (HMO) patients, and $26.5 \%$ for Medicare Preferred Provider Organization (PPO) patients).

\section{Association of Clinical and Social Complexity with Glucose Control in Diabetics}

The results of the final model including controls for age, gender, CCI, mental/behavioral health diagnoses, and SDI score are presented in Table 2. As the addition of SDI had little effect on CCI and mental/behavioral health variables, we only reported the results of the final model. Full model results are available in Appendix 2. In the OCHIN cohort, CCI scores of 4 or higher were associated with a greater probability of poor glucose control. Conversely, the number of mental and behavioral health diagnoses were inversely associated with the probability of poor glucose control, such that those with 1 or more than 1 mental/behavioral health condition(s) had significantly lower odds of poor glucose control (see Table 2). In the smaller HCN cohort neither the CCI scores nor the number of mental and behavioral health diagnoses were significantly associated with poor glucose control after adjustment for age, gender, and SDI.

Although there was not a significant relationship between CCI and diabetes control across the 2 cohorts, increasing social complexity (as assessed by neighborhood-level SDI score) did have a significant association with higher rates of poor diabetic control in both the OCHIN and HCN cohorts. Even after adjusting for age, gender, CCI, and mental/behavioral health diagnoses, for each 10point increase in the SDI, the odds of poorly controlled diabetes increased by $5 \%$ in the OCHIN cohort and $3 \%$ in the HCN cohort. 


\begin{tabular}{|c|c|c|}
\hline & \multicolumn{2}{|c|}{$\begin{array}{l}\text { Tested Diabetes Cohort } \\
\text { Odds Ratio for Poor Glucose Control } \\
(95 \% \mathrm{CI})\end{array}$} \\
\hline & $\begin{array}{c}\text { OCHIN } \\
\mathrm{N}=63,906\end{array}$ & $\begin{array}{c}\mathrm{HCN} \\
\mathrm{N}=9422\end{array}$ \\
\hline Charlson Comorbidity Index: 0 to 1 & Referent & Referent \\
\hline Charlson Comorbidity Index: 2 to 3 & $0.99(0.94$ to 1.03$)$ & $0.99(0.88$ to 1.11$)$ \\
\hline Charlson Comorbidity Index: 4 to 5 & $1.19(1.12 \text { to } 1.27)^{*}$ & $1.14(0.89$ to 1.45$)$ \\
\hline Charlson Comorbidity Index: $6+$ & $1.12(1.01 \text { to } 1.24)^{*}$ & $0.87(0.67$ to 1.13$)$ \\
\hline Mental/Behavioral Health Diagnoses: 0 & Referent & Referent \\
\hline Mental/Behavioral Health Diagnoses: 1 & $0.91(0.87 \text { to } 0.96)^{*}$ & $1.09(0.97$ to 1.22$)$ \\
\hline Mental/Behavioral Health Diagnoses: more than 1 & $0.81(0.73 \text { to } 0.90)^{*}$ & $0.97(0.78$ to 1.20$)$ \\
\hline Social Deprivation Index ${ }^{\dagger}$ & $1.05(1.04 \text { to } 1.06)^{*}$ & $1.03(1.01 \text { to } 1.06)^{*}$ \\
\hline
\end{tabular}

Results of full logistic regression model of the odds of poor diabetes control, controlling for age, gender, Charlson Comorbidity Index category, number of mental and behavioral health diagnoses and Social Deprivation Index (SDI) score of the patient's census tract $(\mathrm{OCHIN})$ or zip code tabulation area $(\mathrm{HCN})$. SDI scores were calculated using national percentile ranks of the SDI. Diabetic patients were defined as those with at least one HbA1c measurement in 2015. Poor control was defined as HbA1c $>9$ at the last test in 2015 .

*Significant at the .05 level.

${ }^{\dagger}$ Odds ratio for an increase of 1 unit (10 national percentile ranks) in score.

CI, confidence interval; OCHIN, Oregon Community Health Information Network; HCN, Health Choice Network.

\section{Association of Clinical and Social Complexity on Provider Quality Metrics}

We also evaluated how adjusting primary care providers' patient panels for clinical and social complexity impacted the assessment of provider quality metrics for glucose control. After adjusting for CCI, our estimates suggest that $95.3 \%$ of OCHIN providers and $87.7 \%$ of $\mathrm{HCN}$ providers would improve their performance assessment (ie, the percent of diabetics in poor control) by 1 or more percentage points (Table 3). Adding an adjustment for mental/ behavioral health diagnoses to the CCI did not result in additional improvement in provider quality metrics. However, adjusting for SDI in addition to CCI and mental/behavioral health diagnoses improved the assessed rates of glucose control by an additional one percentage point or more for $15.5 \%$ of OCHIN providers and $7.1 \%$ of $\mathrm{HCN}$ providers. Finally, because the median SDI of both safety net cohorts is higher than would be found in the general population, we determined the predicted adjustment for safety net providers if the SDI score for their patient panels was set to the national median of 50 . Notably, we found that $75 \%$ of providers across the OCHIN and $\mathrm{HCN}$ safety net cohorts would expect to see an improvement in their performance assessment of 1 percentage point or more if their panel had an SDI equivalent to the national median $(25 \%$ would see a
$1-2 \%$ improvement, $45 \%$ would see a $2-5 \%$ improvement, and $5 \%$ would see more than a $5 \%$ improvement in their assessed performance metrics).

\section{Discussion}

To our knowledge, this is the first study to examine the combined impact of social and clinical complexity on the odds of poor diabetes control and to assess the potential implications for provider quality metrics. Our findings suggest that community-level social complexity, as assessed through census or ZCTA-level SDI score, had a significant positive association with the odds of poor glucose control in both the OCHIN and HCN cohorts. Across provider panels, including adjustment for social complexity and clinical complexity improved the assessment of diabetes performance metrics more than adjusting for clinical complexity alone. These findings support existing evidence of the influence of community-level social risk factors on diabetes metrics, ${ }^{6,39,42-45}$ and are consistent with previous studies that have found an association between provider quality measures and neighborhood-level characteristics of their patient populations. ${ }^{14,46,47}$

The effect of clinical complexity was less consistent across the 2 cohorts. Within the OCHIN cohort, a CCI of over 4 was associated with greater odds of poor diabetes control. Although a CCI of 6 


\begin{tabular}{|c|c|c|}
\hline & \multicolumn{2}{|c|}{$\begin{array}{l}\text { Poor Glucose Control } \\
\text { in Diabetics }\end{array}$} \\
\hline & OCHIN & $\mathrm{HCN}$ \\
\hline Number of Providers & 898 & 114 \\
\hline \multicolumn{3}{|c|}{$\begin{array}{l}\text { Providers with improved performance assessment after adding adjustment } \\
\text { for Charlson Index to adjustment for age and sex }\end{array}$} \\
\hline 1 to 2 percentage point improvement & $227(25.3 \%)$ & $66(57.9 \%)$ \\
\hline 2 to 5 percentage point improvement & $616(68.6 \%)$ & $33(28.9 \%)$ \\
\hline Greater than 5 percentage point improvement & $13(1.4 \%)$ & $1(0.9 \%)$ \\
\hline \multicolumn{3}{|c|}{$\begin{array}{l}\text { Providers with improved performance assessment after adding adjustment } \\
\text { for Mental and Behavioral Health Score to adjustment for age, sex and } \\
\text { Charlson Index }\end{array}$} \\
\hline 1 to 2 percentage point improvement & 0 & 0 \\
\hline 2 to 5 percentage point improvement & 0 & 0 \\
\hline Greater than 5 percentage point improvement & 0 & 0 \\
\hline \multicolumn{3}{|c|}{$\begin{array}{l}\text { Difference in provider metric due to adding adjustment for SDI score to } \\
\text { age, sex, Charlson Index and MHBH Score }\end{array}$} \\
\hline 1 to 2 percentage point improvement & $113(12.6 \%)$ & $6(5.3 \%)$ \\
\hline 2 to 5 percentage point improvement & $25(2.8 \%)$ & $2(1.8 \%)$ \\
\hline Greater than 5 percentage point improvement & 0 & 0 \\
\hline \multicolumn{3}{|c|}{$\begin{array}{l}\text { Difference in provider metric due to adding adjustment to the median } \\
\text { National SDI score to adjustment for age, sex, Charlson Index and } \\
\text { MHBH Score }\end{array}$} \\
\hline 1 to 2 percentage point improvement & $213(23.7 \%)$ & $49(43.0 \%)$ \\
\hline 2 to 5 percentage point improvement & $421(46.9 \%)$ & $34(29.8 \%)$ \\
\hline Greater than 5 percentage point improvement & $54(6.0 \%)$ & $3(2.6 \%)$ \\
\hline
\end{tabular}

Following the CMS procedure for adjustment of Performance Metrics for panel clinical complexity, provider quality metrics were adjusted for providers with more than 20 patients with the outcome.

OCHIN, Oregon Community Health Information Network; HCN, Health Choice Network; MHBH, Mental/Behavioral Health Diagnoses; SDI, Social Deprivation Index.

or more was also associated with greater odds of poor diabetes control, the odd ratio was slightly smaller than for a CCI of 4 to 5 . Interestingly, having 1 or more mental/behavioral health diagnoses was associated with reduced odds of poor control. Previous research has found a similar association between higher levels of clinical complexity and better measures of diabetes control. ${ }^{48-50}$ A potential explanation for this trend is that patients with more comorbidities use health care more frequently and thus may be more closely monitored. ${ }^{51}$ Given that those with mental/behavioral health conditions are likely to have more frequent touch points with the health care system overall, a similar explanation could also underlie the finding that those with more than 1 mental/behavioral health diagnoses had reduced odds of poor diabetes control relative to those with no mental/behavioral health diagnoses.

Within the HCN cohort, measures of clinical complexity and mental/behavior health diagnoses were not significant. A potential reason for this could be the relatively small sample size (there were only $9422 \mathrm{HCN}$ patients, compared with 63,906 OCHIN patients), which is also reflected in the number of patients in the higher CCI categories ( 724 for $\mathrm{HCN}$ compared with 10,500 for OCHIN). It could also be due to other unobserved differences between these 2 cohorts that are beyond those scope of the present analysis. Future studies should investigate further these inconsistencies and explore the association between clinical and social complexity and diabetes control in additional cohorts of patients.

Our findings have significant clinical, health systems, and policy implications. First, these findings suggest that providers caring for populations with greater social complexity may benefit from having their performance metrics and reimbursements adjusted. As the United States moves increasingly toward value-based purchasing models of reimbursement, further research and refinement of a combined measure of social complexity may contribute to more effective and equitable value-based 
models that incentivize the care of socially complex patients. The use of value-based models unadjusted for social complexity may financially penalize providers that serve vulnerable populations, ultimately resulting in provider reluctance to serve these patients and fewer care options among those individuals that need the most care. However, even with appropriate adjustment for social complexity, providers caring for socially complex populations may also need additional infrastructure and supports in place to address the needs of the patients they serve. Thus, any social risk adjustment approach should be carefully implemented to ensure that the need for funding to support infrastructure among safety net providers is not minimized.

Perhaps more importantly, these findings reinforce the vital importance of understanding and addressing $\mathrm{SDH}$ as a core component of strategies to reduce disparities and improve health outcomes across the US. Indeed, the association between increasing SDI and poorer diabetic control lends further support to the importance of primary care-public health integration efforts to address patient- and neighborhood-level social conditions to improve health care quality and utilization. Moreover, these findings underscore the need for continued patientlevel social risk screening both to identify patients with unmet needs and to inform decisions about individual patient care. ${ }^{52}$

\section{Limitations}

Several limitations must be considered when interpreting our results. First, in the absence of robust patient-level SDH data, we utilized aggregate community-level data about where patients live as a measure of social complexity. As more patient-level social risk screening data becomes available, future research should examine the concordance between measures of community- and patient-level social risk factors and seek to understand the appropriate uses of both sources of information. ${ }^{53}$ Such research could help to disentangle whether community-level data are a valid a proxy for information on patient-reported social risks, as well as enhance our understanding of the relative impact of "place"-or the neighborhood and community environments in which people live-on both individual and population health, independent of individual-level risk factors. ${ }^{54}$

Second, our study examined already socially vulnerable cohorts, so the effect size of social complexity adjustment may underestimate the true impact of SDH on diabetes control. Future research in this area should expand to include more heterogeneous populations, such as national or nonsafety net samples, to examine the impact of social complexity across a broader spectrum of patients. Similarly, there are limitations of the sample cohort for unbiased assessment of the impact of social complexity on provider performance. The CMS formula multiplies the ratio of observed/expected performance by the sample mean to get the adjusted performance; because our study cohorts have higher degrees of social complexity than the national population, adjusting to the sample mean is adjusting to a mean that is likely higher than a national sample.

\section{Conclusions}

In the absence of wide-spread patient-reported social determinants of health data, we used an areabased measure of social deprivation to understand the impact of social complexity on provider performance metrics. Even after controlling for clinical complexity, our findings showed a positive association between higher levels of community-level social deprivation and the odds of poor diabetes control. As payment models shift toward valuebased pay, it will be important to adjust for social complexity when assessing differences in provider metric scores.

To see this article online, please go to: http://jabfm.org/content/ 33/4/600.full.

\section{References}

1. Shipman SA, Sinsky CA. Expanding primary care capacity by reducing waste and improving the efficiency of care. Health Aff (Millwood) 2013;32: 1990-7.

2. O'Malley AS, Rich EC, Maccarone A, DesRoches CM, Reid RJ. Disentangling the linkage of primary care features to patient outcomes: a review of current literature, data sources, and measurement needs. J Gen Intern Med 2015;30:576-85.

3. Porter ME, Larsson S, Lee TH. Standardizing patient outcomes measurement. $\mathrm{N}$ Engl J Med 2016;374:504-6.

4. Braveman P, Gottlieb L. The social determinants of health: it's time to consider the causes of the causes. Public Health Rep 2014;129:19-31.

5. Commission on Social Determinant of Health. Closing the gap in a generation: health equity through action on the social determinants of health. 
Commission on Social Determinants of Health final report. Geneva, Switzerland: World Health Organization; 2008.

6. Tarasuk V, Cheng J, de Oliveira C, Dachner N, Gundersen C, Kurdyak P. Association between household food insecurity and annual health care costs. CMAJ 2015;187:E429-E436.

7. Havranek EP, Mujahid MS, Barr DA, et al. Social determinants of risk and outcomes for cardiovascular disease: a scientific statement from the american heart association. Circulation 2015;132:873-98.

8. Solbu MD, Thomson PC, Macpherson S, et al. Serum phosphate and social deprivation independently predict all-cause mortality in chronic kidney disease. BMC Nephrol 2015;16:194.

9. Hong CS, Atlas SJ, Chang Y, et al. Relationship between patient panel characteristics and primary care physician clinical performance rankings. JAMA 2010;304:1107-13.

10. Bailey SR, O'Malley JP, Gold R, Heintzman J, Likumahuwa S, DeVoe JE. Diabetes care quality is highly correlated with patient panel characteristics. J Am Board Fam Med 2013;26:669-79.

11. Landon BE, O'Malley AJ, Keegan T. Can choice of the sample population affect perceived performance: implications for performance assessment. J Gen Intern Med 2010;25:104-9.

12. Paddison C, Elliott M, Parker R, et al. Should measures of patient experience in primary care be adjusted for case mix? Evidence from the English General Practice Patient Survey. BMJ Qual Saf 2012;21:634-40.

13. Tyo KR, Gurewich D, Shepard DS. Methodological challenges of measuring primary care delivery to pediatric Medicaid beneficiaries who use community health centers. Am J Public Health 2013;103:273-5.

14. Chien AT, Wroblewski K, Damberg C, et al. Do physician organizations located in lower socioeconomic status areas score lower on pay-for-performance measures? J Gen Intern Med 2012;27:548-54.

15. O'Connor PJ, Bodkin NL, Fradkin J, et al. Diabetes performance measures: current status and future directions. Diabetes Care 2011;34:1651-9.

16. Adler NE, Stead WW. Patients in context-EHR capture of social and behavioral determinants of health. N Engl J Med 2015;372:698-701.

17. Gottlieb L, Sandel M, Adler NE. Collecting and applying data on social determinants of health in health care settings. JAMA Intern Med 2013;173: 1017-20.

18. Rosenthal MB. Beyond pay for performanceEmerging models of provider-payment reform. $\mathrm{N}$ Engl J Med 2008;359:1197-200.

19. Charlson ME, Charlson RE, Peterson JC, Marinopoulos SS, Briggs WM, Hollenberg JP. The Charlson comorbidity index is adapted to predict costs of chronic disease in primary care patients. J Clin Epidemiol 2008;61:1234-40.

20. DeVoe JE, Bazemore AW, Cottrell EK, et al. Perspectives in primary care: a conceptual framework and path for integrating social determinants of health into primary care practice. Ann Fam Med 2016;14:104-8.

21. Center for Medicare \& Medicaid Services. Accountable Health Communities Model. https:// innovation.cms.gov/initiatives/ahcm/. Accessed February 17, 2017.

22. Committee on the Recommended Social and Behavioral Domains and Measures for Electronic Health Records. Capturing social and behavioral domains and measures in electronic health records: Phase 2. 2014. https://www.nap.edu/catalog/18951/ capturing-social-and-behavioral-domains-andmeasures-in-electronic-health-records.

23. National Association of Community Health Centers. Protocol for Responding to and Assessing Patients' Assets, Risks, and Experiences (PRAPARE). 2017. http://www.nachc.org/research-and-data/prapare/.

24. Gold R, Bunce A, Cowburn S, et al. Adoption of social determinants of health EHR tools by community health centers. Ann Fam Med 2018;16:399-407.

25. Gold R, Cottrell E, Bunce A, et al. Developing electronic health record (EHR) strategies related to health center patients' social determinants of health. J Am Board Fam Med 2017;30:428-47.

26. Cottrell E, Dambrun K, Cowburn S, et al. Variation in EHR documentation of social determinants of health across a national network of community health centers. Am J Prevent Care 2019;57:S65-S73.

27. Cottrell EK, Dambrun K, Cowburn S, et al. Variation in electronic health record documentation of social determinants of health across a national network of community health centers. Am J Prev Med 2019;57:S65-S73.

28. Bazemore AW, Cottrell EK, Gold R, et al. "Community vital signs": incorporating geocoded social determinants into electronic records to promote patient and population health. J Am Med Inform Assoc 2016;23:407-12.

29. Fleming BB, Greenfield S, Engelgau MM, Pogach LM, Clauser SB, Parrott MA. The Diabetes Quality Improvement Project: moving science into health policy to gain an edge on the diabetes epidemic. Diabetes Care 2001;24:1815-20.

30. Pogach L, Aron DC. Sudden acceleration of diabetes quality measures. JAMA 2011;305:709-10.

31. National Academies of Sciences E, and Medicine. Accounting for Social Risk Factors in Medicare Payment: Identifying Social Risk Factors. Washington, DC: The National Academies Press; 2016.

32. Devoe JE, Gold R, Spofford M, et al. Developing a network of community health centers with a common electronic health record: description of the Safety Net West Practice-Based Research Network 
(SNW-PBRN). J Am Board Fam Med 2011;24: 597-604.

33. Devoe JE, Sears A. The OCHIN community information network: bringing together community health centers, information technology, and data to support a patient-centered medical village. J Am Board Fam Med 2013;26:271-8.

34. Centers for Medicare \& Medicaid Services. 2014 Physician Quality Reporting System (PQRS) Measure Specifications Manual for Claims and Registry Reporting of Individual Measures. 2013.

35. Charlson M, Szatrowski TP, Peterson J, Gold J. Validation of a combined comorbidity index. J Clin Epidemiol 1994;47:1245-51.

36. Di Iorio B, Cillo N, Cirillo M, De Santo NG. Charlson Comorbidity Index is a predictor of outcomes in incident hemodialysis patients and correlates with phase angle and hospitalization. Int J Artif Organs 2004;27:330-6.

37. Mandelberg JH, Kuhn RE, Kohn MA. Epidemiologic analysis of an urban, public emergency department's frequent users. Acad Emergency Med 2000;7:637-46.

38. Walker ER, McGee RE, Druss BG. Mortality in mental disorders and global disease burden implications: a systematic review and meta-analysis. JAMA Psychiatry 2015;72:334-41.

39. Butler DC, Petterson S, Phillips RL, Bazemore AW. Measures of social deprivation that predict health care access and need within a rational area of primary care service delivery. Health Serv Res 2013;48:539-59.

40. Robert Graham Center. Social Deprivation Index (SDI). 2018. https://www.graham-center.org/rgc/ maps-data-tools/sdi/social-deprivation-index.html.

41. Medicare.gov. The Official US Government Site for Medicare: Home Health Compare statistical methods. 2018; https://www.medicare.gov/home healthcompare/Data/statistical-methods.html.

42. Fitzpatrick T, Rosella LC, Calzavara A, et al. Looking beyond income and education: socioeconomic status gradients among future high-cost users of health care. Am J Prev Med 2015;49:161-71.

43. Cournane S, Conway R, Byrne D, O'Riordan D, Coveney S, Silke B. Social deprivation and the rate of emergency medical admission for older persons. QJM 2016;109:645-51.

44. Agardh E, Allebeck P, Hallqvist J, Moradi T, Sidorchuk A. Type 2 diabetes incidence and socioeconomic position: a systematic review and metaanalysis. Int J Epidemiol 2011;40:804-18.

45. Strom JL, Egede LE. The impact of social support on outcomes in adult patients with type 2 diabetes: a systematic review. Curr Diab Rep 2012;12:769-81.

46. Lines LM, Rosen AB, Ash AS. Enhancing administrative data to predict emergency department utilization: the role of neighborhood sociodemographics. J Health Care Poor Underserved 2017; 28:1487-508.

47. Fiscella K, Franks P. Impact of patient socioeconomic status on physician profiles: a comparison of census-derived and individual measures. Med Care 2001;39:8-14.

48. Woodard LD, Landrum CR, Urech TH, Wang D, Virani SS, Petersen LA. Impact of clinical complexity on the quality of diabetes care. Am J Manag Care 2012;18:508-14.

49. Woodard LD, Urech T, Landrum CR, Wang D, Petersen LA. Impact of comorbidity type on measures of quality for diabetes care. Med Care 2011;49:605-10.

50. Min LC, Wenger NS, Fung C, et al. Multimorbidity is associated with better quality of care among vulnerable elders. Med Care 2007;45:480-8.

51. Higashi T, Wenger NS, Adams JL, et al. Relationship between number of medical conditions and quality of care. N Engl J Med 2007;356:2496504.

52. National Academies of Sciences E, and Medicine. Integrating social care into the delivery of health care: Moving upstream to improve the nation's health. Washington, DC: The National Academies Press; 2019.

53. Gottlieb LM, Francis DE, Beck AF. Uses and misuses of patient- and neighborhood-level social determinants of health data. Perm J 2018;22: $18-78$.

54. Diez Roux AV. Investigating neighborhood and area effects on health. Am J Public Health 2001; 91:1783-9. 


\section{Appendix 1: Mental and Behavioral Health Condition Coding}

Psychosis or bipolar disorder

ICD-9 diagnosis codes: 295, 295.0, 295.00, 295.01, 295.02, 295.03, 295.04, 295.05, 295.1, 295.1, 295.11, 295.12, 295.13, 295.14, 295.15, 295.2, 295.20, 295.21, 295.22, 295.23, 295.24, 295.25, 295.3, 295.30, 295.31, 295.32, 295.33, 295.34, 295.35, 295.4, 295.40, 295.41, 295.42, 295.43, 295.44, 295.45, 295.5, 295.50, 295.51, 295.52, 295.53, 295.54, 295.55, 295.6, 295.60, 295.61, 295.62, 295.63, 295.64, 295.65, 295.7, 295.70, 295.71, 295.72, 295.73, 295.74, 295.75, 295.8, 295.80, 295.81, 295.82, 295.83, 295.84, 295.85, 295.9, 295.90, 295.91, 295.92, 295.93, 295.94, 295.95, 296.0, 296.00, 296.01, 296.02, 296.03, 296.04, 296.05, 296.06, 296.1, 296.10, 296.11, 296.12, 296.13, 296.14, 296.15, 296.16, 296.4, 296.40, 296.41, 296.42, 296.43, 296.44, 296.45, 296.46, 296.5, 296.50, 296.51, 296.52, 296.53, 296.54, 296.55, 296.56, 296.6, 296.60, 296.61, 296.62, 296.63, 296.64, 296.65, 296.66, 296.7, 296.8, 296.80, 296.81, 296.89, 297.0, 297.1, 297.2, 297.3, 301.22, V11.0

ICD-10 diagnosis codes: F20, F20.0, F20.1, F20.2, F20.3, F20.5, F20.8, F20.81, F20.89, F20.9, F21, F22, F23, F24, F25, F25.0, F25.1, F25.8, F25.9, F28, F29, F30, F30.1, F30.10, F30.11, F30.12, F30.13, F30.2, F30.3, F30.4, F30.8, F30.9, F31, F31.0, F31.1, F31.10, F31.11, F31.12, F31.13, F31.2, F31.3, F31.30, F31.31, F31.32, F31.4, F31.5, F31.6, F31.60, F31.61, F31.62, F31.63, F31.64, F31.7, F31.70, F31.71, F31.72, F31.73, F31.74, F31.75, F31.76, F31.77, F31.78, F31.8, F31.81, F31.89, F31.9

Substance abuse

ICD-9 diagnosis codes: 291, 291.0, 291.1, 291.2, 291.3, 291.4, 291.5, 291.8, 291.81, 291.82, 291.89, 291.9, 292.0, 292.1, 292.11, 292.12, 292.2, 292.8, 292.81, 292.82, 292.83, 292.84, 292.85, 292.89, 292.9, 303, 303.0, 303.00, 303.01, 303.02, 303.03, 303.9, $303.90,303.91,303.92,303.93,304,304.0,304.00,304.01,304.02,304.03,304.1,304.10,304.11,304.12,304.13,304.2,304.20$, 304.21 , 304.22, 304.23, 304.3, 304.30, 304.31, 304.32, 304.33, 304.4, 304.40, 304.41, 304.42, 304.43, 304.5, 304.50, 304.51, 304.52, 304.53, 304.6, 304.60, 304.61, 304.62, 304.63, 304.7, 304.70, 304.71, 304.72, 304.73, 304.8, 304.80, 304.81, 304.82, $304.83,304.9,304.90,304.91,304.92,304.93,305,305.0,305.00,305.01,305.02305 .03,305.1,305.2,305.20,305.21,305.22$, $305.23,305.3,305.30,305.31,305.32,305.33,305.4,305.40,305.41,305.42,305.43,305.5,305.50,305.51,305.52,305.53$, 305.6, 305.60, 305.61, 305.62, 305.63, 305.7, 305.70, 305.71, 305.72, 305.73, 305.8, 305.80, 305.81, 305.82, 305.83, 305.9, $305.90,305.91,305.92,305.93$

ICD-10 diagnosis codes: F10.2, F10.20, F10.21, F10.22, F10.220, F10.221, F10.229, F10.23, F10.230, F10.231, F10.232, F10.239, F10.24, F10.25, F10.250, F10.251, F10.259, F10.26, F10.27, F10.28, F10.280, F10.281, F10.282, F10.288, F10.29, F11.2, F11.20, F11.21, F11.22, F11.220, F11.221, F11.222, F11.229, F11.23, F11.24, F11.25, F11.250, F11.251, F11.259, F11.28, F11.281, F11.282, F11.288, F11.29, F13.2, F13.20, F13.21, F13.22, F13.220, F13.221, F13.229, F13.23, F13.230, F13.231, F13.232, F13.239, F13.24, F13.25, F13.250, F13.251, F13.259, F13.26, F13.27, F13.28, F13.280, F13.281, F13.282, F13.288, F13.29, F14.2, F14.20, F14.21, F14.22, F14.220, F14.221, F14.222, F14.229, F14.23, F14.24, F14.25, F14.250, F14.251, F14.259, F14.28, F14.280, F14.281, F14.282, F14.288, F14.29, F15.20, F18.10, F18.12, F18.120, F18.121, F18.129, F18.14, F18.15, F18.150, F18.151, F18.159, F18.17, F18.18, F18.180, F18.188, F18.19, F18.2, F18.20, F18.21, F18.22, F18.220, F18.221, F18.229, F18.24, F18.25, F18.250, F18.251, F18.259, F18.27, F18.28, F18.280, F18.288, F18.29, F19.2, F19.20, F19.21, F19.22, F19.220, F19.221, F19.222, F19.229, F19.23, F19.230, F19.231, F19.232, F19.239, F19.24, F19.25, F19.250, F19.251, F19.259, F19.26, F19.27, F19.28, F19.280, F19.281, F19.282, F19.288, F19.29

Patients were classified as having a history of depression, psychosis or bipolar disorders, or substance abuse based on presence of the diagnosis codes in their electronic health records. they were then classified as having 0,1 , or more than one of these three types of conditions. 


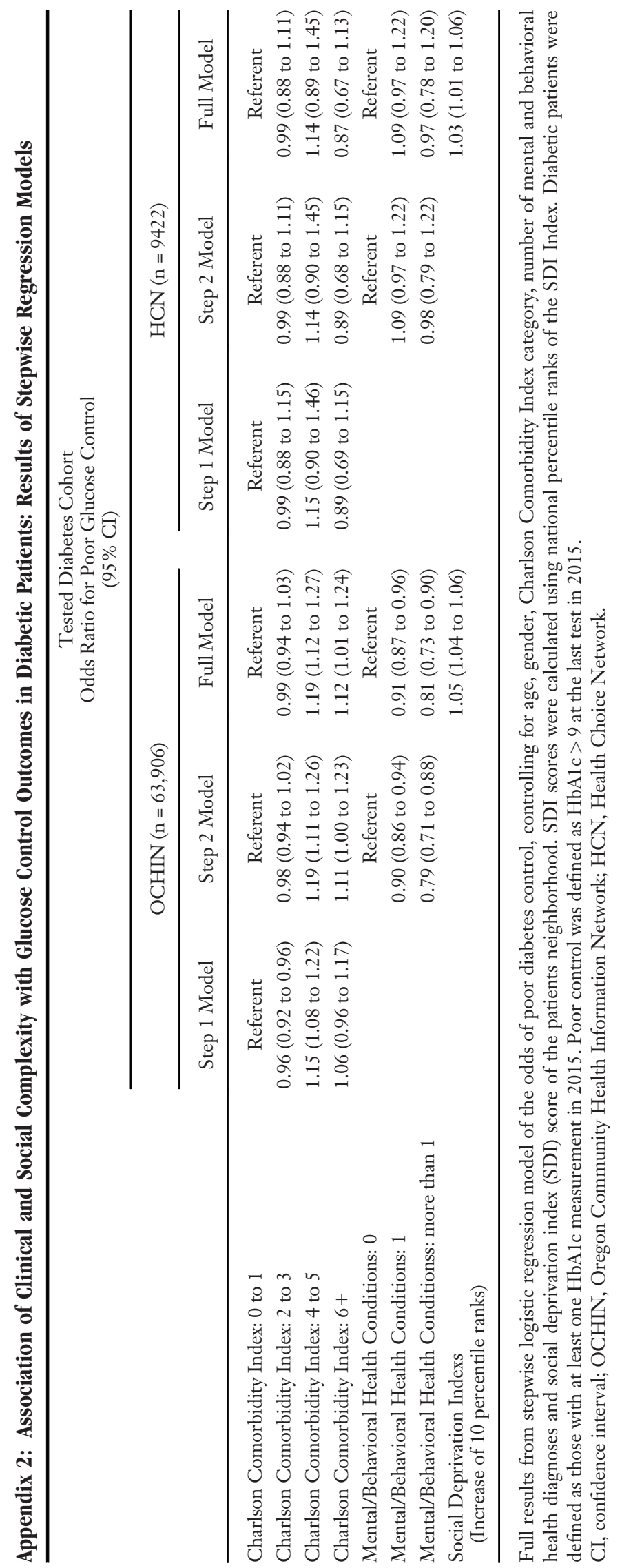

